\title{
La prothèse provisoire fixée par technique directe : une solution d'urgence
}

Provisional fixed partial denture with direct technique: an emergency solution

\section{MOTS-CLEFS :}

- Prothèse provisoire, technique directe, temporisation

\section{KEYWORDS:}

- Provisional restoration, direct technique, temporization
AOS 2014;269:10-15

DOI: $10.1051 / \mathrm{aos} / 2014303$

(C) EDP Sciences 2014

\section{Résumé}

A l'exception de certaines restaurations prothétiques partielles (bridge collé, facette), tout traitement prothétique passe impérativement par une étape de temporisation au moyen de prothèses provisoires. Celles-ci, remplissant plusieurs rôles, peuvent être réalisées aussi bien au laboratoire qu'au cabinet dentaire.

$\mathrm{Si}$ les techniques indirectes (au laboratoire) garantissant une intégration optimale de la prothèse provisoire, sont actuellement les techniques les plus préconisées et utilisées, elles restent inadaptées pour certaines situations cliniques. En effet, dans des cas d'urgence, le praticien a recours aux techniques directes de réalisation des prothèses provisoires de façon extemporanée, en l'attente des prothèses de temporisation du laboratoire. Ces techniques directes, toujours d'actualité, répondent également parfaitement aux exigences de toute restauration prothétique.

\author{
Abstract
}

Except for some partial prosthetic restorations (bonded fixed partial denture, lumineers), for each prosthetic treatment an imperative step of temporization is set by provisional restorations. Latter, fulfilling several roles, can be performed both in the laboratory and at the dental office.

While indirect techniques (laboratory), ensuring optimal integration of the temporary prosthesis, are currently the most recommended and used techniques, they are still inapropriate for certain clinical situations. Indeed, in emergencies, the dentist uses direct techniques of making provisional prothesis extemporaneously, pending laboratory provisional restorations. These direct techniques, still current, also fully meet the requirements of any prosthetic restoration.

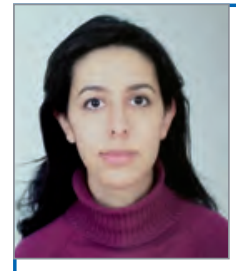

- Layla ASSILA, Chirurgien-dentiste, spécialiste et PhDc au service de prothèse conjointe. Faculté de médecine dentaire, Rabat, Maroc.

Loubna EL FIGUIGUI, Chirurgien-dentiste, spécialiste et PhDc au service de prothèse conjointe. Faculté de médecine dentaire, Rabat, Maroc.

Hicham SOUALHI, Professeur assistant au service de prothèse conjointe. Chirurgien-dentiste, résidente et PhDc au service de prothèse conjointe. Faculté de médecine dentaire, Rabat, Maroc.

Amal EL YAMANI, Professeur d'enseignement supérieur, chef de service de prothèse conjointe. Faculté de médecine dentaire, Rabat, Maroc. 
$\mathrm{L}^{\mathrm{a}}$ a prothèse provisoire est considérée comme une étape primordiale incontournable parmi les séquences successives du plan de traitement en prothèse fixée. À l'exception des préparations pelliculaires pour bridges collés et pour facettes, toute restauration prothétique est précédée de cette étape dite de « temporisation $"$.

Pour remplir son rôle sur les plans esthétique, fonctionnel et biologique, la prothèse provisoire est généralement réalisée au laboratoire selon des techniques indirectes. Ces techniques présentent de meilleures qualités, garantes d'une meilleure intégration de la prothèse.

Cependant, en cas d'urgence esthétique, fonctionnelle ou biologique (fracture ou perte de prothèse au niveau d'un secteur esthétique...), l'élaboration de la prothèse provisoire par technique directe trouve parfaitement son indication. Elle est alors réalisée entièrement au cabinet dentaire de façon extemporanée selon des techniques directes (dans l'attente des prothèses provisoires de laboratoire).

Malgré l'évolution des techniques et des matériaux, la technique directe est toujours d'actualité, répondant parfaitement aux exigences de toute restauration prothétique.

À travers des cas cliniques, nous illustrons l'apport de ces différentes techniques directes de réalisation de prothèse provisoire.

Quelles sont ces techniques directes, et quels sont leurs avantages et leurs limites?

\section{RÔLES DE LA PROTHÈSE PROVISOIRE FIXÉE [1-4]}

La prothèse provisoire remplit plusieurs rôles et fonctions qui contribuent à la réussite de la prothèse définitive.

Sur le plan fonctionnel, le remplacement des dents absentes et/ou le recouvrement des dents délabrées permet le rétablissement des différentes fonctions orales. Aussi la prothèse permet-elle de figer la situation clinique sur le plan occlusal, en préservant une stabilité des rapports dento-dentaires intra- et inter-arcades initiaux ou en permettant l'acquisition d'une nouvelle position occlusale correcte.

Par la même occasion, une stabilité de la situation parodontale est obtenue en évitant toute prolifération tissulaire marginale au niveau des limites cervicales, et en jouant un rôle thérapeutique dans la cicatrisation du parodonte profond dans certains cas.

Le rôle biologique de la prothèse provisoire concerne aussi bien la protection des dents piliers contre toute agression physique, mécanique, chimique, thermique ou bactérienne, que la protection du parodonte marginal contre toute irritation mécanique ou réaction inflammatoire (suite à la rétention alimentaire principalement).

Sur le plan esthétique, la prothèse provisoire permet de maintenir ou de restaurer l'esthétique des dents (antérieures notamment), et elle représente un moyen de communication entre patient, praticien et prothésiste, sous la forme d'un croquis qui préfigure les prothèses définitives et qui doit être validé par le patient.

Outre les rôles précités et qui concernent la prothèse provisoire en général, celle réalisée par technique directe présente l'avantage de pouvoir être utilisée comme un traitement d'urgence en cas de besoin, notamment dans le secteur antérieur où il est question de préjudice esthétique.

\section{CRITÈRES D'UNE PROTHÈSE PROVISOIRE DE QUALITÉ $[1,2,5]$}

Une prothèse provisoire réussie, remplissant les rôles précités, doit répondre à certains critères morphologiques, indépendamment de la technique d'élaboration utilisée :

I adaptation cervicale ;

$\checkmark$ dégagement des embrasures ;

$\checkmark$ rétablissement correct des points de contact ;

$\checkmark$ adaptation ou intégration occlusale ;

$\checkmark$ morphologie axiale correcte ;

$\checkmark$ état de surface poli et lisse.

\section{TECHNIQUES DIRECTES DE L'ÉLABORATION DE LA PROTHÈSE PROVISOIRE FIXÉE}

Les techniques directes de réalisation de la prothèse provisoire au cabinet dentaire sont au nombre de trois :

\les coques préformées ;

$\checkmark$ la technique d'iso-moulage ;

$\checkmark$ la block-technique.

Le matériau le plus utilisé pour réaliser la prothèse provisoire au cabinet dentaire est la résine autopolymérisable. Cependant, il existe également des coffrets de coques préfabriquées métalliques (pour les dents postérieurs), en composite photopolymérisable ou en polycarbonates pour les dents antérieures $[2,3,6]$. Ces matériaux doivent avoir une esthétique acceptable avec une stabilité de leur teinte dans le temps, et être biocompatibles, faciles à manipuler, résistants à l'usure, compatibles avec les autres matériaux dentaires et faibles conducteurs thermiques $[2,3,6,7]$. 


\section{Coques préformées $[1,6,8]$}

Cette technique utilise un coffret de couronnes préfabriquées. Elles sont en polycarbonate pour les dents antérieures, et en métal ou composite malléable photopolymérisable pour les dents postérieures.

La coque est choisie selon le diamètre mésio-distal existant entre les dents adjacentes, et adaptée par la suite sur les préparations.

\section{Description (fig. 1)}

1. Choix de la coque selon le diamètre mésio-distal de la dent concernée.

2. Essayage et adaptation marginale.
3. Rebasage de la coque à la résine, réalisé ensuite pour une meilleure adaptation cervicale et une rétention adéquate.

4. Élimination des excès.

5. Essayage en bouche et vérification de l'occlusion.

6. Ajustage occlusal et polissage.

\section{Avantages}

I Réalisation rapide : sans nécessité de wax-up ou d'empreinte préalable.

I Facilité de mise en œuvre.

I Bonnes propriétés mécaniques et bon état de surface du matériau constituant la coque.
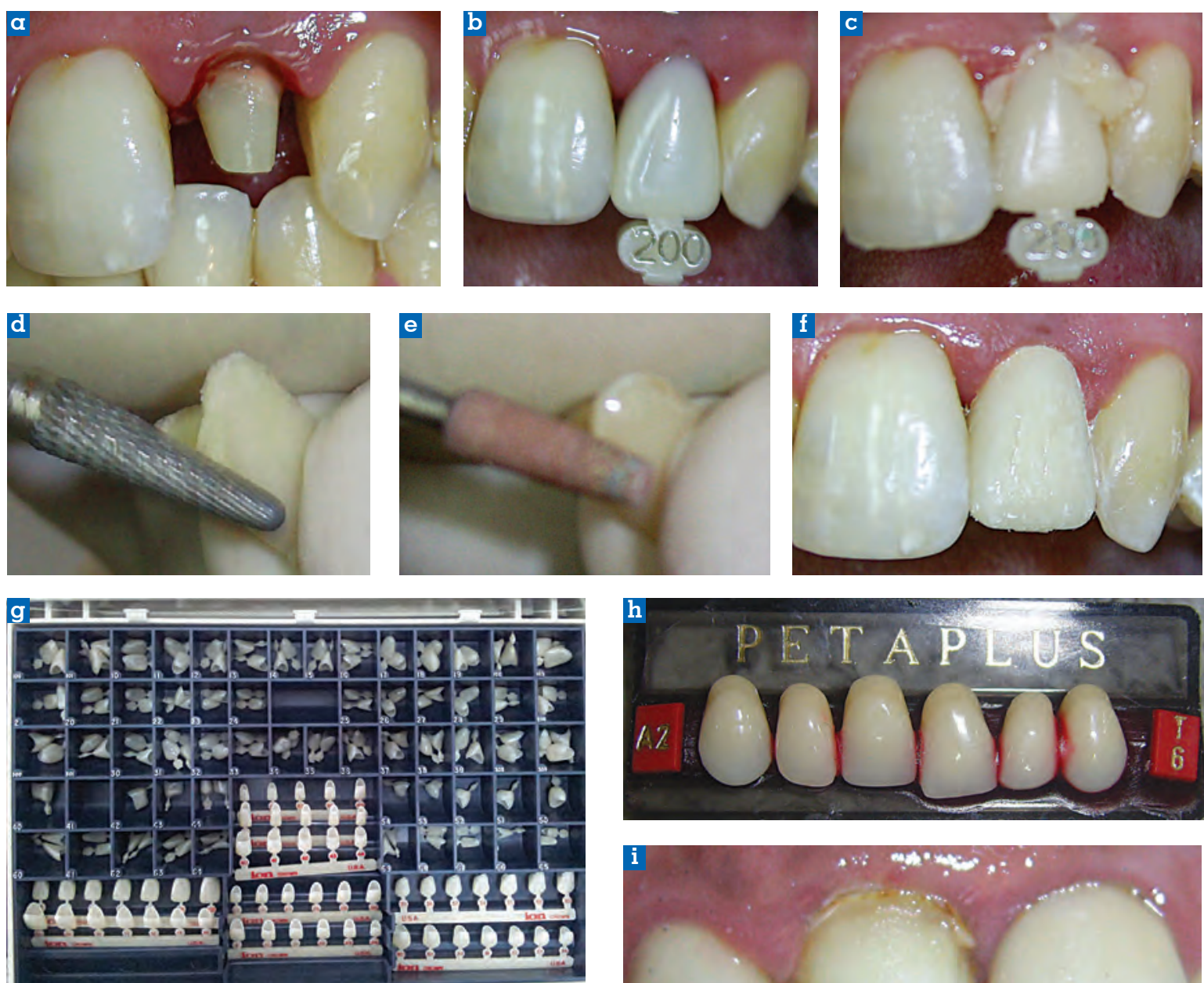

$\measuredangle$ Fig. 1 : Coques préformées.

a. Situation clinique initiale : la 22 nécessitant une restauration provisoire.

b. Choix et essayage de la coque préformée.

c. Rebasage de la coque préformée à la résine autopolymérisable.

d. Élimination des excès et ajustage cervical.

e. Finition de la prothèse provisoire ajustée.

f. Prothèse provisoire scellée en bouche.

g. Coffret de coques préformées en polycarboxylate.

h. Jeu de dents de prothèse adjointe. Choix de la dent selon la teinte, la forme et la dimension adéquates.

i. Taille périphérique sur la 11 nécessitant une prothèse provisoire.

j. Adaptation de la dent évidée et rebasée à la résine sur la préparation.
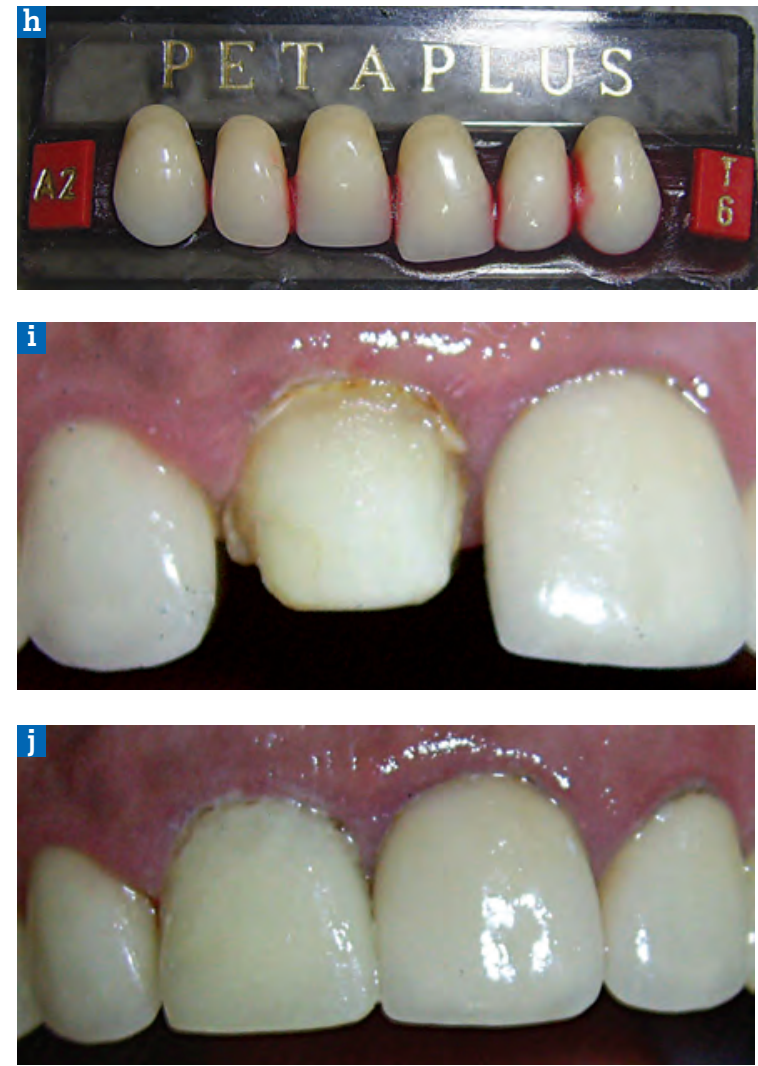

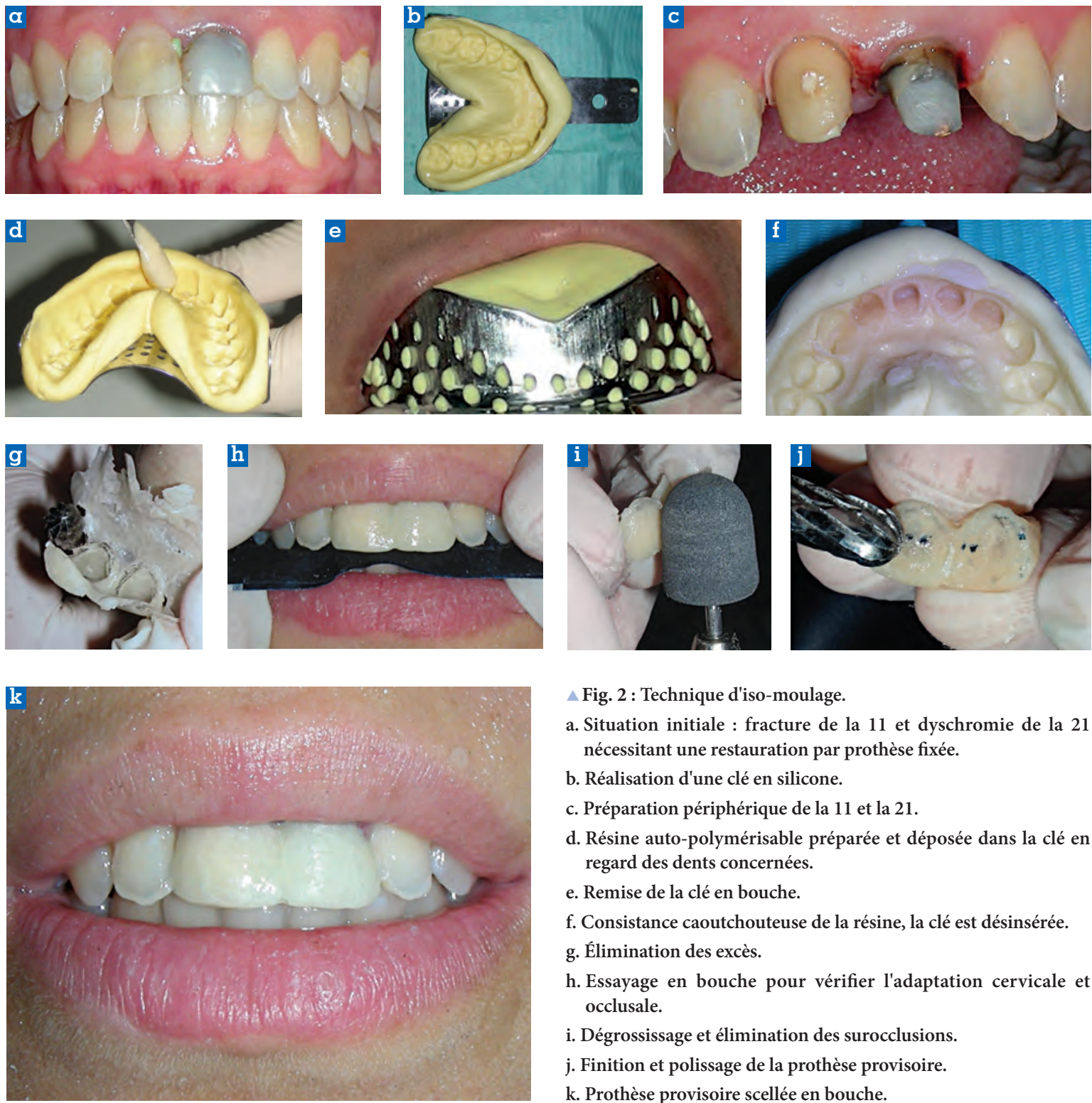

$\triangle$ Fig. 2 : Technique d'iso-moulage.

a. Situation initiale : fracture de la 11 et dyschromie de la 21 nécessitant une restauration par prothèse fixée.

b. Réalisation d'une clé en silicone.

c. Préparation périphérique de la 11 et la 21.

d. Résine auto-polymérisable préparée et déposée dans la clé en regard des dents concernées.

e. Remise de la clé en bouche.

f. Consistance caoutchouteuse de la résine, la clé est désinsérée. g. Élimination des excès.

h. Essayage en bouche pour vérifier l'adaptation cervicale et occlusale.

i. Dégrossissage et élimination des surocclusions.

j. Finition et polissage de la prothèse provisoire.

k. Prothèse provisoire scellée en bouche.

I Polyvalence : cette technique peut être utilisée pour une couronne simple, une dent à tenon ou les éléments de bridge.

I Forme anatomique correcte : elle permet une rapidité et une facilité de réalisation.

I Esthétique satisfaisante pour les dents antérieures.

\section{Limites}

I Ajustage occlusal important, parfois enlevant complètement l'épaisseur de la coque.

1 Adaptation parfois difficile de la forme de la coque.

I Esthétique nulle pour les dents postérieures (coiffes métalliques).

1 Prix de la boîte.

I Nécessité d'un stock important.

Cette technique peut également utiliser des dents destinées à la prothèse adjointe, ce qui permet d'avoir un choix plus vaste en teintes, formes et dimensions (fig. 1, h à j).

\section{TECHNIQUE D'ISO-MOULAGE [1, 8, 9]}

Cette technique nécessite la confection d'une clé en silicone directement en bouche (avant la préparation) ou sur le modèle d'étude éventuellement modifié par wax-up (si la dent en question est délabrée).

La prothèse provisoire est réalisée en injectant ou en remplissant la clé avec de la résine auto-polymérisable.

\section{Description (fig. 2)}

1. Réalisation de la clé (en bouche ou sur modèle d'étude).

2. Préparation périphérique de la dent.

3. Résine auto-polymérisable préparée et déposée dans la clé en regard de la (ou des) dent(s) concernée(s).

4. Repositionnement de la clé en bouche sur le moignon isolé.

5. Dès que la consistance de la résine est caoutchouteuse, des mouvements de va-et-vient verticaux 

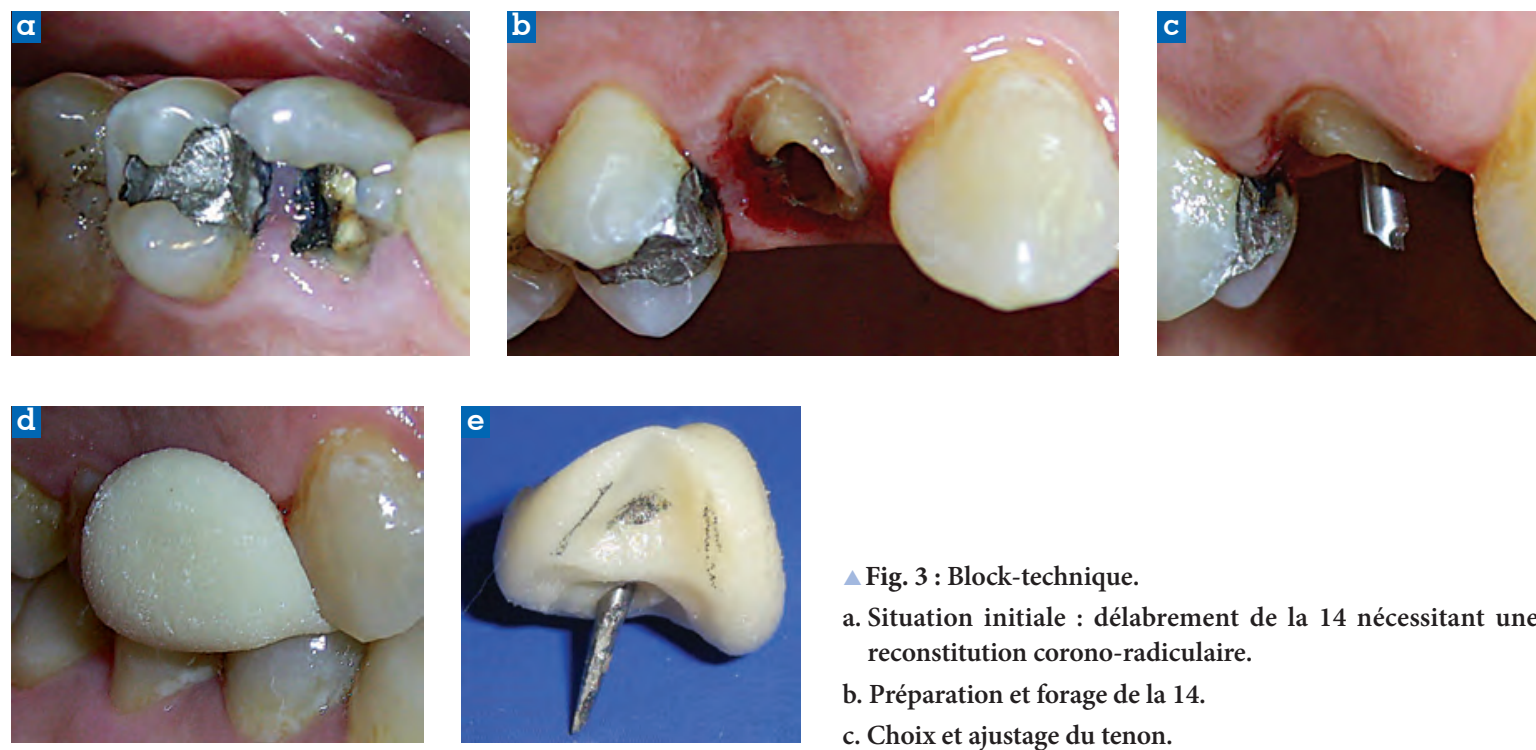

$\triangle$ Fig. 3 : Block-technique.

a. Situation initiale : délabrement de la 14 nécessitant une reconstitution corono-radiculaire.

b. Préparation et forage de la 14 .

c. Choix et ajustage du tenon.
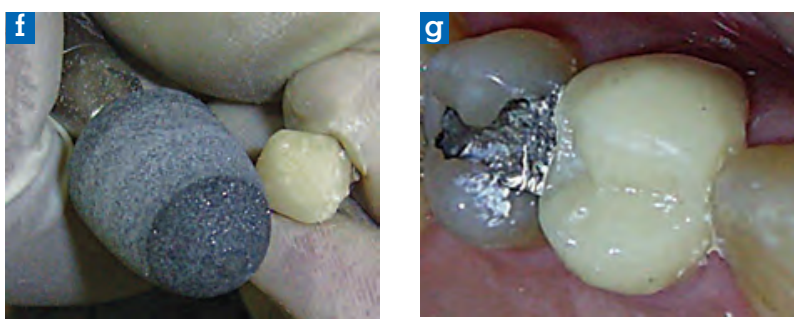

d. Une boule de résine de consistance pâteuse est déposée sur la dent concernée et le patient est invité à fermer en OIM.

e. Désinsertion de la boule de résine. À ce stade, elle n'a aucune forme anatomique. Une morphologie correcte est recréée en respectant les limites de la préparation et les repères occlusaux et par une sculpture des faces axiales et proximales. Les retouches occlusales sont minimes.

f. Finition et polissage de la prothèse provisoire.

g. Prothèse provisoire scellée en bouche.

sont exécutés afin de contrecarrer la contraction de polymérisation.

6. Élimination des excès.

7. Essayage en bouche pour vérifier l'adaptation cervicale et occlusale.

8. Dégrossissage jusqu'à obtention d'une adaptation marginale correcte, et élimination des surocclusions.

9. Polissage de la prothèse provisoire.

\section{Avantages}

I Restitution d'une morphologie adaptée grâce à l'utilisation de la clé.

I Facilité de mise en œuvre.

Ajustages occlusaux minimes.

I Esthétique correcte.

I Technique économique.

\section{Limites}

\ Fragilité en cas de restaurations de grande étendue. \Étape diagnostique préliminaire parfois nécessaire (en cas de délabrement important).

\section{BLOCK-TECHNIQUE $[1,8,10]$}

C'est l'une des techniques les moins décrites, mais elle n'en demeure pas moins excellente, notamment pour les secteurs cuspidés. Elle nécessite peu de matériel, et est réalisée à partir de résine chémopolymérisable. Elle consiste à modeler une anatomie coronaire à partir d'une quantité déterminée de résine malaxée sous forme d'une boule (unitaire) ou d'un bourrelet (bridge de 3 éléments).

\section{Description (fig. 3)}

1. Après la réalisation ou la reprise de la préparation de la dent, la boule (ou le bourrelet) de résine à consistance pâteuse est placée en bouche et est adaptée directement sur la préparation.

2. On demande au patient de fermer en OIM, et on lui fait faire les mouvements mandibulaires afin de modeler la face occlusale de la future prothèse, durant la polymérisation.

3. À ce stade, la couronne n'a pas de forme anatomique. On désinsère alors la boule de résine qui porte l'enregistrement des points et trajets des cuspides antagonistes, ainsi que les limites de la préparations en son intrados. Une morphologie correcte est recréée à l'aide d'une fraise à résine montée sur pièce à main, en respectant les données fonctionnelles, ainsi que les formes anatomiques des faces proximales et bombées des faces axiales.

4. Un rebasage pour parfaire l'adaptation cervicale peut s'avérer nécessaire à ce stade.

5. On réalise à la fin un polissage à l'aide d'une cupule à résine. 


\section{Avantages}

ISimplicité : cette technique ne nécessite pas de matériel spécifique.

Inatomie occlusale : elle est d'emblée fonctionnelle et ne nécessite pas de rectifications.

I Possibilité de réaliser un bridge (cela dépend de la dextérité du praticien).

\section{Limites}

Difficulté : il faut une certaine expérience et une habitude de manipulation.

I Esthétique faible.

I Anatomie sommaire qui reste imprécise en la comparant aux autres techniques.

$\checkmark$ Réalisation longue : elle nécessite un temps de manipulation plus long pour une sculpture anatomique des différentes faces de la prothèse.

\section{CONCLUSION}

La prothèse provisoire fixée offre une restauration des différentes fonctions orales et une réhabilitation de l'esthétique tout en préservant l'intégrité dentaire et parodontale.

Les techniques directes de réalisation permettent d'obtenir une prothèse provisoire selon des méthodes faciles, simples, et surtout rapides et efficaces, dans des situations d'urgence, tout en répondant aux exigences cliniques.

Chacune de ces techniques directes représente une approche thérapeutique qui doit être choisie et adaptée selon la situation clinique.

\section{Bibliographie}

[1] Lucas S, Ghrenassia Ch, Esclassan R, Guyonnet JJ. La temporisation en prothèse fixée. Stratégie prothétique 2008;8(2):97-104

[2] Viennot S, Malquarti G, Guiu C, Pirel C. Prothèse fixée de temporisation. Encycl Méd Chir (Elsevier, Paris) Odontologie 2007;23-272-B-20.

[3] Gratton DG, Aquilino SA. Interim restorations. Dent Clin N Am 2004;48:487-497.

[4] Laurent M, Aboudharam G, Laborde G. Prothèse transitoire : à propos d'une technique. Clinic 1999;20(7):435-439.

[5] Christensen GJ. Provisional restorations for fixed prosthodontics. J Am Dent Assoc 1996;127:249-252.

[6] Malquarti G, Comte B, Allard Y, Martin JP, Bois D. Prothèse immédiate provisoire. Encycl Méd Chir (Elsevier, Paris) Odonologie 1998;23-272-B-10.

[7] Meyer JM, Belser U. Les matériaux pour couronnes et ponts provisoires. Réalités Cliniques 1994;5(1):15-24.

[8] Marmy O, Cioppi G, Michelini F. Restaurations provisoires. Cah Proth 1996;96:45-52.

[9] Farre P, Guyonnet JJ, Girard P. Couronne provisoire, technique originale d'iso-moulage en occlusion. Cah Proth 2001;115:65-68.

[10] Sous M. Prothèse fixée transitoire « la blocktechnique ». Cah Proth 2002;118:45-48.

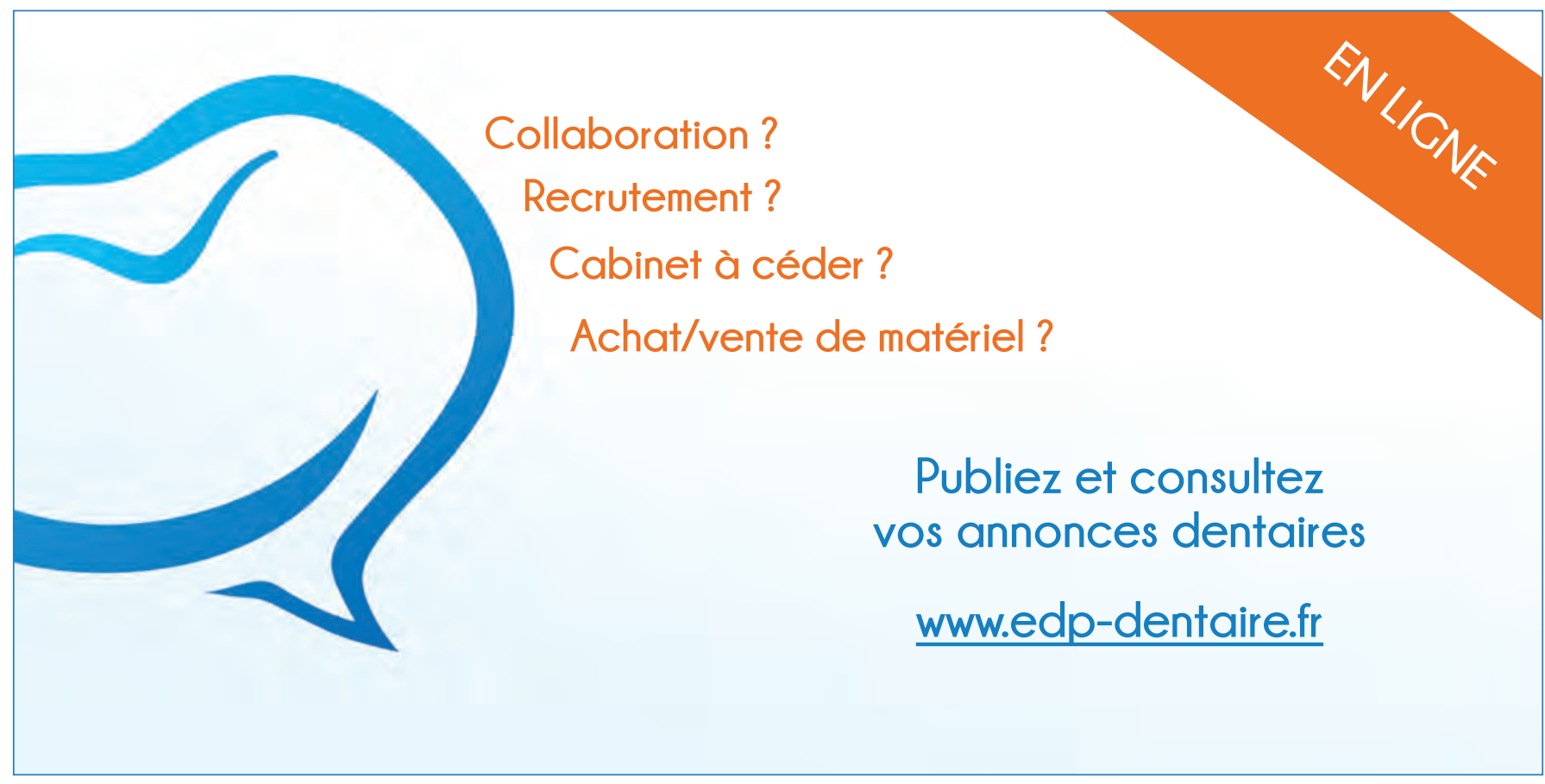

